\section{Tacrolimus as an Effective and Safe Therapeutic Alternative in Vernal Keratoconjunctivitis Resistant to Conventional Treatment}

González-Medina $\mathrm{M}^{1}$, Blasco-Valero $\mathrm{C}^{1,3}$, Martín-Begué $\mathrm{N}^{2}$, VilàIndurain $\mathrm{B}^{1,3}$, Garriga-Baraut $\mathrm{T}^{1,3}$

${ }^{1}$ Unidad de Alergología Pediátrica, Hospital Universitario Materno-Infantil Vall d'Hebron, Barcelona, Spain

${ }^{2}$ Unidad de Oftalmología Pediátrica, Hospital Universitario Materno-Infantil Vall d'Hebron, Barcelona, Spain

${ }^{3}$ Grup Creixement i Desenvolupament, Institut de Recerca Vall d'Hebron, Barcelona, Spain

J Investig Allergol Clin Immunol 2018; Vol. 28(5): 345-346 doi: $10.18176 /$ jiaci.0282

Key words: Vernal keratoconjunctivitis. Tacrolimus. Calcineurin inhibitors. Ocular allergy. Steroids.

Palabras clave: Queratoconjuntivitis vernal. Tacrolimus. Inhibidores de la calcineurina. Alergia ocular. Corticosteroides.

Vernal keratoconjunctivitis (VKC) is a severe and chronic form of ocular allergy that can cause severe visual complications [1]. The estimated prevalence of VKC in Europe is $3.2 / 10000$, whereas it is almost endemic in subtropical countries [2]. VKC is classified as an $\operatorname{IgE}$ and non-IgE-mediated ocular allergy in the most recent European guidelines. IgE-mediated hypersensitivity can be assessed through skin prick testing, serum-specific IgE measurements, and, if necessary, conjunctival provocation testing [3]. As for pathogenesis, a significantly high count of neutrophils, lymphocytes, and eosinophils on the ocular surface has been reported [4]. The diagnosis is based on the clinical history and suggestive signs and symptoms. However, no single clinical feature can confirm the diagnosis. VKC is characterized by periods of improvement and exacerbations of symptoms. Flareups can be triggered either by re-exposure to the allergen or, more frequently, by nonspecific stimuli such as sunlight, wind, and dust. Useful options for pharmacologic therapy include topical antihistamines [5], mast cell stabilizers, and doubleaction drugs. Topical corticosteroids should be administered in short, pulsed regimens when the cornea is involved [6]. Calcineurin inhibitors are off-label treatments in the European Union. In contrast, in Japanese studies, immunosuppressive eye drops are added before corticosteroids in moderate or more severe cases where antiallergic eye drops alone are not sufficiently effective [7]. Cyclosporine has been widely used to treat mild-to-severe VKC, although up to $10 \%$ of patients have a poor response or, in some cases, are totally unresponsive [8]. Topical tacrolimus has been reported to be effective and safe in the short term for severe VKC resistant to topical cyclosporine. However, treatment with topical tacrolimus in the pediatric population in our geographic area has received little attention.

We present the results of a retrospective study performed at the Pediatric Allergy Unit of Vall d'Hebron University
Hospital, Barcelona, Spain to evaluate the usefulness and safety of topical ocular treatment with tacrolimus $0.03 \%$ ointment in pediatric patients affected by VKC refractory to conventional treatment.

A cross-sectional retrospective study was performed between January 2009 and May 2017. Patients were evaluated in the Pediatric Allergy Unit or the Pediatric Ophthalmology Department. The diagnosis of VKC was based on published guidelines. Refractoriness was defined as persistence of signs and symptoms despite conventional treatment. Clinical ocular signs and symptoms were collected. Sensitization to airborne allergens was also evaluated. The statistical evaluation was performed using IBM SPSS Statistics for Windows, Version 23.0 (IBM Corp). Data are presented as median, mean (SD), and/or range. To determine the association between variables of interest, the Wilcoxon matched-pairs test was used. A $P$ value of $<.05$ was considered statistically significant.

The study population included 17 patients (12 males, 5 females), with a mean age of 12 years, and a mean age at onset of symptoms of 6 years. A family history of atopy was identified in $53 \%$ of patients. Fifteen patients $(88 \%)$ were sensitized to airborne allergens (house dust mites, $66.7 \%$; pet dander, $20 \%$; pollens, $6.7 \%$; and molds, $6.6 \%$ ). The patients were provided with $0.03 \%$ tacrolimus eye ointment and instructed to apply a rice grain-sized amount in the affected eye twice daily in addition to conventional treatment. The time tacrolimus was administered depended on the duration of the flare, with discontinuation as early as possible, when the condition had resolved. Patients received treatment with tacrolimus for a mean of 21 days (11.3 days). On average, the flare-up lasted 56 days (range, 0-112 days) before initiation of tacrolimus. However, once tacrolimus was started, this period was reduced to 18 days (range, 7-56) $(P<.05)$.

Given the difficulty comparing the decrease in the dose of oral and topical antihistamines and corticosteroids (tablets, syrup, and/or drops) according to age and weight, the decrease in the use of oral and topical antihistamines and oral or topical corticosteroids was evaluated according to days of need for antihistamines and corticosteroids. Nine patients required oral and topical antihistamines for a mean of 336 days a year before starting treatment with tacrolimus. These were stopped completely after initiation of tacrolimus in 8 patients $(P<.05)$. In the only patient in whom the treatment could not be stopped, the number of days decreased by $50 \%$ (168 days per year) $(P<.05)$. However, the use of lubricants remained unchanged.

The mean time that patients needed corticosteroids before starting tacrolimus was 168 days (17.8 days) a year. However, tacrolimus reduced this period to a mean of 90 days (16.8 days) a year $(P<.05)$. Moreover, in 2 patients, tacrolimus enabled topical corticosteroids to be discontinued. The mean number of flare-ups per year was not reduced after initiating tacrolimus (6 per year). However, the duration of each exacerbation was reduced, as was severity, which enabled doses to be decreased or combined corticosteroid eye drops to be discontinued. Our data support the corticosteroid-sparing effect of tacrolimus and the reduction in the frequency of adverse effects due to long-term corticosteroids. Moreover, a considerable improvement in symptoms was seen with tacrolimus. These data were collected using a visual analog scale (VAS), which 


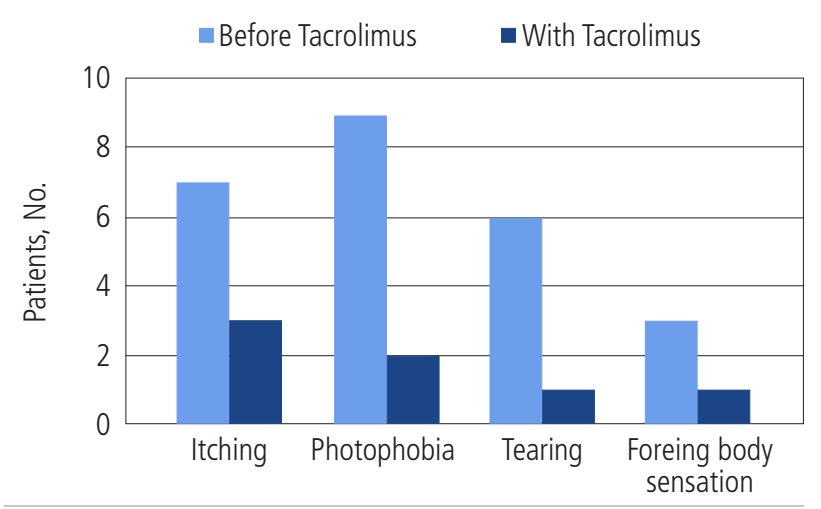

Figure. Improvement in clinical symptoms with tacrolimus.

was combined with the self-assessment provided by the patient and the clinical impression of improvement perceived by the parents. The symptoms evaluated were itching, photophobia, tearing, and foreign body sensation. The mean VAS score before tacrolimus was $3.8(0.70)$, which fell to 2.5 (0.28) after therapy with tacrolimus $(P<.05$, Figure $)$. As for adverse effects with tacrolimus, only 1 patient reported a burning sensation.

In the light of these findings, we propose low-dose topical tacrolimus $0.03 \%$ ointment as a valid therapeutic alternative in pediatric patients with $\mathrm{VKC}$ that is refractory to conventional treatment. This approach makes it possible to significantly reduce time on topical corticosteroids and thus render therapy more tolerable for most patients. Data from Japanese guidelines indicate that this treatment should be included in EU guidelines.

\section{Funding}

The authors declare that no funding was received for the present study.

\section{Conflicts of Interest}

The authors declare that they have no conflicts of interest.

\section{Previous Presentation}

Data from this report were presented in poster format at the Pediatric Allergy and Asthma Meeting - PAAM 2017, held in London, October 26-28, 2017.

\section{References}

1. Leonardi A. Vernal keratoconjunctivitis: pathogenesis and treatment. Prog Ret Eye Res. 2002;21:319-39.

2. Bremond-Gignac D, Donadieu J, Leonardi A, Pouliquen P, Doan S, Chiambarretta $F$, et al. Prevalence of vernal keratoconjunctivitis: a rare disease? $\mathrm{Br} J$ Ophthalmol. 2008;92:1097-102.

3. Sánchez MC, Fernández Parra B, Matheu V, Navarro A, Ibáñez MD, Dávila I, et al. Allergic Conjunctivitis. J Investig Allergol Clin Immunol. 2011;21,Suppl 2:1-19.

4. Hu Y, Matsumoto Y, Dogru M, Okada N, Igarashi A, Fukagawa $K$, et al. The differences of tear function and ocular surface findings in patients with atopic keratoconjunctivitis and vernal keratoconjunctivitis. Allergy. 2007;62:917-25.

5. A del Cuvillo, Sastre J, Montoro J, Jáuregui I, Dávila I, Ferrer M. Allergic Conjunctivitis and H1 Antihistamines. J Investig Allergol Clin Immunol. 2009;19 Suppl 1:11-8.

6. Sánchez-Hernández MC, Montero J, Rondon C, Benítez del Castillo JM, Velázquez E, Herreras JM, et al. Consensus Document on Allergic Conjunctivitis (DECA). J Investig Allergol Clin Immunol. 2015;25(2):94-106.

7. Takamura E, Uchio E, Ebihara N, Ohno S, Ohashi Y, Okamoto $S$, et al. Japanese guidelines for allergic conjunctival diseases 2017. Allergology International. 2017;66(2):220-9.

8. Pucci N, Caputo R, di Grande L, de Libero C, Mori F, Barni S, et al. Tacrolimus vs. cyclosporine eyedrops in severe cyclosporineresistant vernal keratoconjunctivitis: A randomized, comparative, double-blind, crossover study. Pediatr Allergy Immunol. 2015;26:256-61.

Manuscript received February 7, 2018; accepted for publication June 11, 2018.

\author{
Mónica González Medina \\ Passeig Vall d'Hebron 119-129 \\ 08035 Barcelona, Spain \\ E-mail:monicagonzmedina@gmail.com
}

